\title{
Radon-induced surface contaminations in low background experiments
}

\author{
M. Clemenza, C. Maiano, L. Pattavina ${ }^{\text {a }}$, E. Previtali \\ Università degli Studi Milano-Bicocca and I.N.F.N. Sezione Milano-Bicocca, Milan, Italy
}

Received: 9 October 2011 / Published online: 18 November 2011

(C) The Author(s) 2011. This article is published with open access at Springerlink.com

\begin{abstract}
In low background experiments the reduction of all possible radioactive contaminants is a crucial point for detector construction. This is also true for the surface contaminants, either those introduced during the production of detector components or those introduced during handling, treatment or storage. One of the most critical issue in this field is the control of the contamination induced by ${ }^{222} \mathrm{Rn}$ and its progenies in the environment where the detectors are assembled and stored. Radioactive atoms can stick on detector components and create a net increase of the contaminants present on their surfaces, introducing an additional-often not negligible - source of background. The reduction of this kind of contaminations can become of primary importance in the case of fully sensitive devices, like cryogenic particle detectors. In this paper the analysis on the Rn sticking factor for copper and tellurium dioxide-the two main materials used for the construction of the CUORE detector-is discussed. The diffusion of radioactive atoms inside the detector components is considered in order to evaluate the effective contribution of $\mathrm{Rn}$ exposure to the background counting rate of an experiment.
\end{abstract}

\section{Introduction}

During last decades strong efforts were spent to project and built new generation low background experiments, such as those dedicated to Double Beta Decay (DBD) [1-4] and Dark Matter (DM) [5-8] searches. To be able to achieve the required, extremely low, counting rates these experiments are located deep underground and use specially designed active or passive shields for minimizing the external background. Finally, they adopt very stringent criteria for material radio-purity $[9,10]$. In particular, material selection is no more limited to the sensitive components of the detector as

a e-mail: luca.pattavina@mib.infn.it it was in the past-but it is extended to detector supports and shields as well as to all the ancillaries that can be so close to the detector to contribute to its final background. Moreover, the successful achievement of extremely low levels of contaminations in material bulk has strongly increased the significance of surface contaminations. These are due to radioisotopes present in the environment that stick on the surfaces of materials during the detector assembly. The main sources of this kind of contamination are usually isotopes belonging to the $U$ and $T h$ natural chains and in particular ${ }^{222} \mathrm{Rn}$ and its progenies.

Surface contaminations are particularly critical for fully active detectors (namely devices without a surface dead layer), like cryogenic particle detectors (or bolometers). In these devices low range particles like betas, alphas and nuclear recoils emitted either by the detector surface or by the surfaces of nearby components, can reach the active volume producing a background signal [11, 12]. For example, in the case of the CUORE experiment the bolometers are $\mathrm{TeO}_{2}$ crystals held in vacuum by a copper structure. The experiment aims at the detection of neutrinoless DBD of ${ }^{130} \mathrm{Te}$ that should produce a monochromatic signal, distinguishable from the background only by its characteristic energy $(2530 \mathrm{keV})$. An alpha particle emitted by ${ }^{210}$ Po (one of the more common environmental contaminants), that decays with a $Q$ value of $5.4 \mathrm{MeV}$ can mimic a DBD signal whenever it deposits the right fraction of energy in the detector (namely about $2530 \mathrm{keV}$ ). This happens either in the case where the decay is originated on the detector surface, or in the case where the particle is emitted by a material directly facing the detector since in both cases only a fraction of the total energy of the decay is deposited inside the detector active volume. On the contrary if ${ }^{210} \mathrm{Po}$ is inside the detector bulk the alpha particle is fully contained and generates a signal at the $Q$ value of the decay, too high in energy to be erroneously identified as a DBD.

In principle also beta active isotopes have a similar behavior, namely they provide an important contribution to 
background only when present on detector surfaces of a fully active device. However, while all the alpha particles emitted by the natural chains ( $\mathrm{U}$ and $\mathrm{Th}$ ) have energies high enough to contribute to CUORE DBD background, only 2 isotopes from the same chains emit-in few percent of the decays - a beta particle with a sufficiently high energy to reach the DBD region of interest. On the contrary, betas and nuclear recoils contributed in the low energy (DM) region.

\section{Surface contaminations}

Surface contaminations created during the production phase (e.g. machining, handling, storage and cleaning) of the detector components may be removed by properly studied mechanical or chemical treatments. Special procedures can be studied to avoid the creation of these contaminations. However-even when any possible care in the component production and cleaning have been applied-it is impossible to avoid the exposure of the detector parts to air (or to an atmospheric gas) during the assembly phase and storage of the detector. It is clear that the possible presence in such atmosphere of radioactive isotopes must be carefully considered because this is a source of re-contamination for any previously cleaned item. The effort necessary to have a completely controlled atmosphere during the assembly phase are generally not negligible and for this reason it is necessary to clearly evaluate at which level the presence of such contaminations becomes critical for the final background performances of the detector.

The main source of atmospheric (air) contamination comes from ${ }^{222} \mathrm{Rn}$ and its daughters. This isotope is a ${ }^{238} \mathrm{U}$ daughter and it can emanate from materials where the ${ }^{238} \mathrm{U}$ chain. The re-contamination of materials exposed to a contaminated atmosphere is not produced by ${ }^{222} \mathrm{Rn}$ isotope only but also by its progeny (see Fig. 1). ${ }^{222} \mathrm{Rn}$ daughters emitted in the atmosphere are electrically charged and they can stick on detectors surfaces with a relatively high probability of remaining fixed.

The evaluation of the sticking probability of nuclei on sensitive surfaces of low background experiment components allows to estimate the contribution that an exposure

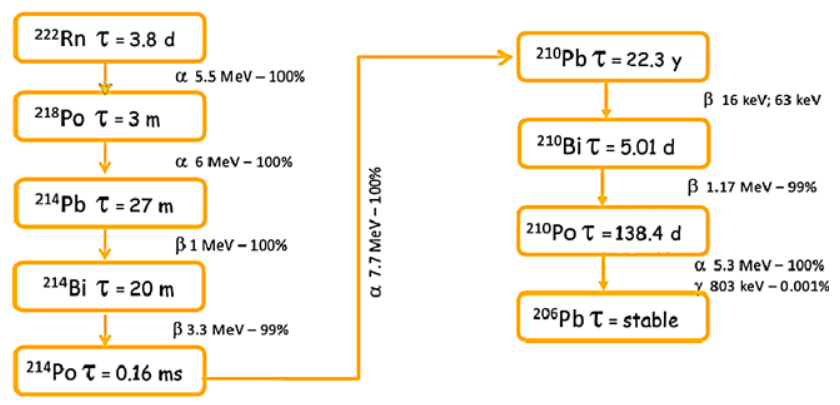

Fig. 1 Alpha and beta decays in the Radon chain to radioactive contaminants will have on the expected sensitivity of the experiment. The values of the sticking factor will allow also to give the minimum requirements that must be fulfilled in order to have a safe environment in which the experiment components can be stored and assembled.

\section{Evaluation of the Radon sticking factor}

In this work, we define the sticking factor $(\Sigma)$ for a nucleus that interacts with a surface as the ratio between the number of nuclei that stick on a surface and the total number of nuclei that are close enough to the surface, less than a $\mathrm{Rn}$ nucleus mean free path, and hence they have a probability different from zero to stick on it:

$\Sigma_{\mathrm{Rn}}=\frac{N(d P=1)}{N(d P \neq 0)}$.

This parameter is related to the Radon concentration, the exposure time and the features of the material surface. In an atmosphere where there are radioactive nuclei, many mechanisms can be identified as possible drivers for surface contaminations. We can define four main processes:

- Deposition of particulate contaminated with radioactive nuclei.

- Diffusion of gaseous components (e.g. Rn) through material surfaces.

- electrostatic attraction between surface and charged nuclei (e.g. ${ }^{222} \mathrm{Rn}$ daughters).

- nuclear recoil implantations related to next-to surface nuclear decay.

In order to be able to study the sticking factor problem a specific environment with a very high Radon concentration was built. Three rocks containing a high ${ }^{238} \mathrm{U}$ concentration were installed inside a Plexiglas box of about $1 \mathrm{~m}^{3}$ tightly closed. In order to avoid possible radioactive deposition of ${ }^{238} \mathrm{U}$ dusts from the sources, a great care was devoted to enclose these rocks in plastic containers that prevent the possible dust dispersion, but enable the Radon emanation.

The time needed to have a stationary concentration of ${ }^{222} \mathrm{Rn}$ inside the box is about 2 weeks. The ${ }^{222} \mathrm{Rn}$ saturation level inside the box is $315 \mathrm{kBq} \mathrm{m}^{-3}$. Obviously the presence of ${ }^{222} \mathrm{Rn}$ in the environment leads to the production of its daughters. Their concentration is related to their half-lives, the shorter is the half-life the smaller will be the concentration: so we expect to have small concentrations of short-living elements (e.g. ${ }^{218} \mathrm{Po}$ and ${ }^{214} \mathrm{Po}$ ) and high concentrations of long-living ones (e.g. ${ }^{210} \mathrm{~Pb}$ and ${ }^{210} \mathrm{Po}$ ). Furthermore, since the we are dealing with a decay chain, after a fixed period of time secular equilibrium takes place: all the elements have the same activities. The time needed to reach this configuration depends on the half-lives of the elements (generally this time is assumed to be $5 \tau_{1 / 2}$ ): for 
the short-living ones a shorter time is needed compared to the long-living ones. The equilibrium of the lower part of the chain will be reached (assuming the ${ }^{210} \mathrm{~Pb}$ activity constant in time) after about 1.5 years, due to the long half-life of ${ }^{210}$ Po (see Fig. 1), in fact the time needed for reaching the equilibrium between two elements depends just on the shorter-living one. Meanwhile the ${ }^{210}$ Po activity will slowly increase trying to reach the equilibrium with its progenitor the ${ }^{210} \mathrm{~Pb}$.

The samples used for the evaluation of the sticking factor are slabs, $50 \times 50 \mathrm{~mm}^{2}$ of surface and $2 \mathrm{~mm}$ thick, of the same materials that will be used in the CUORE experiments [13]: Copper $(\mathrm{Cu})$ and Tellurium dioxide $\left(\mathrm{TeO}_{2}\right)$. In order to minimize the possible influences produced by differences in the surface treatments, all $\mathrm{Cu}$ slabs and $\mathrm{TeO}_{2}$ slabs were respectively processed with the same cleaning procedures. The $\mathrm{TeO}_{2}$ samples were etched in a nitric acid solution while the copper ones with: tumblering, electro-chemical, chemical and magnetron treatments [14]. It is indeed reasonable to assume that the sticking of radioactive elements depends on the effective surface of the sample, different treatments can lead to different surface grindings and thus to different effective surfaces. The tellurium dioxide samples have a grinding RMS of about $10 \mu \mathrm{m}$, while for the copper ones is $20 \mu \mathrm{m}$.

The samples were placed inside the Rn-box using a small access window that does not modify the concentration inside by means of the small access and thus big impedance.

The samples were exposed to Radon (and Radon progenies) contaminations for different period of time. After the exposure to ${ }^{222} \mathrm{Rn}$ the induced contamination on the samples was measured using Silicon surface barrier alpha detectors placing those in a vacuum chamber; the detectors have a $1200 \mathrm{~mm}^{2}$ surface and a dead layer $50 \mathrm{~nm}$ thick.

\section{Sample analysis}

The exposed samples activity was evaluated in order to estimate the effects induced by Radon exposure. In particular there were two main issues that have been considered: the identification of the isotopes that actually sticks on the sample and the dependence of the contamination on the exposure time. The former is obtained studying the equilibrium of the Radon decay chain (namely the activities of the different isotopes belonging to $\mathrm{Rn}$ progenies and their evolution in time after the extraction of the sample from the Rn-box), the latter is studied by activities comparison measured on samples that have undergone a different $\mathrm{Rn}$ exposure.

In the acquired spectra three signals produced by ${ }^{222} \mathrm{Rn}$ daughters were found: ${ }^{218} \mathrm{Po}(6.0 \mathrm{MeV}),{ }^{214} \mathrm{Po}(7.6 \mathrm{MeV})$ and ${ }^{210} \mathrm{Po}(5.3 \mathrm{MeV})$, all these isotopes undergo an alpha decay and in parentheses we have indicated the alpha energy. In Fig. 2 the spectra acquired 3 minutes after the samples extraction from the Rn-box are shown. The signals observed are not compatible with the background (see Fig. 3).

The first important information provided by Fig. 2 is the lack of a clear signal of ${ }^{222} \mathrm{Rn}$ decay: there is no alpha peak at 5.6 MeV. In order to get a stronger evidence for this fact, it is possible to analyze the spectrum acquired at a specific time $t_{x}$, long after the sample extraction from the Rn-box (see Fig. 4). The choice of $t_{x}$ is done in such a way that all the short-living Polonii isotopes are completely decayed $\left(t_{x}>5 \tau_{\mathrm{Po}}\right)$, and the only left over is the ${ }^{222} \mathrm{Rn}$ one (and the ${ }^{210} \mathrm{Po}$ one). In this way the sensitivity to a possible presence of a peak at $5.6 \mathrm{MeV}$ is maximized. Using the sample exposed for the longest time (63 days) it is possible to eval-

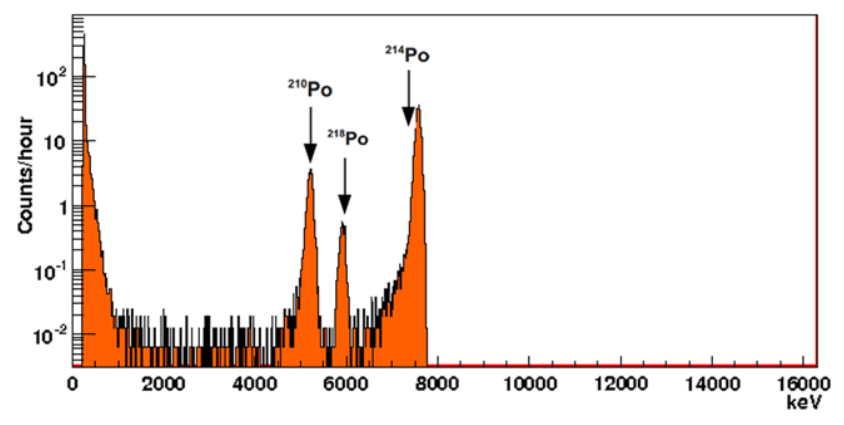

Fig. 2 Acquired spectrum (measurement time $242 \mathrm{~h}$ ) from $0 \mathrm{MeV}$ up to $16 \mathrm{MeV}$ of an exposed copper sample (Copper_1)

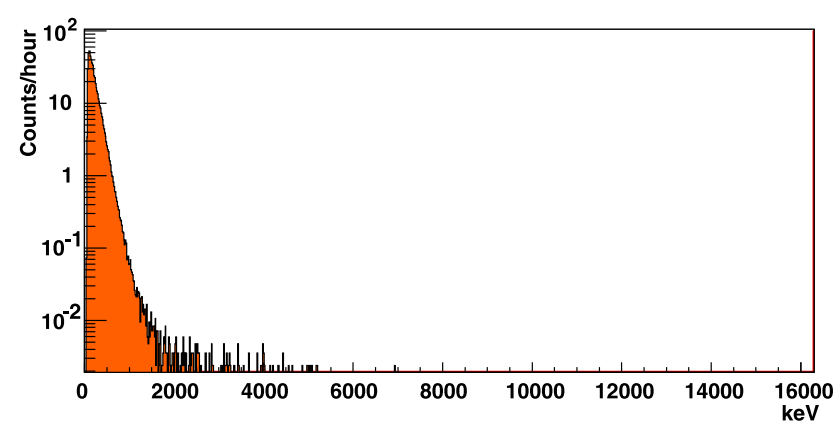

Fig. 3 Background measurement of a copper sample on a SBD detector. The measurement lasted 840 hours

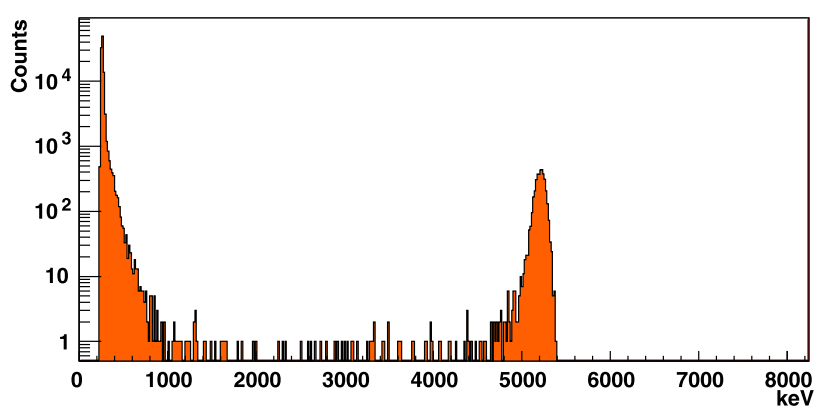

Fig. 4 Copper_ 1 acquired spectrum at $t_{x}=72 \mathrm{~h}$ from the extraction time (measurement time $170 \mathrm{~h}$ ). The only observable signal is the ${ }^{210} \mathrm{Po}$ one 
uate the initial ${ }^{222} \mathrm{Rn}$ contamination $\left(A_{\mathrm{Rn}}^{0}=A_{\mathrm{Rn}}(t=0)\right)$. This is done scaling the activity measured at $t_{x}=72 \mathrm{~h}$ $\left(A_{\mathrm{Rn}}\left(t=t_{x}\right)\right)$ with the decay exponential law: the resulting ${ }^{222} \mathrm{Rn}$ contamination at the beginning of the measurement is $A_{\mathrm{Rn}}^{0}<0.22 \mu \mathrm{Bq} \mathrm{cm}^{-2}$.

The same sample has an initial ${ }^{218}$ Po activity which is much higher, being:

$A_{218 \mathrm{Po}}^{0}=0.15 \pm 0.02 \mathrm{~Bq} \mathrm{~cm}^{-2}$

Moreover the activity of this isotope is observed to decrease with the typical exponential decay rate of ${ }^{218} \mathrm{Po}$. This behavior is consistent with the fact that there is no ${ }^{222} \mathrm{Rn}$ contamination on the sample, hence there is no mechanism that produces ${ }^{218} \mathrm{Po}$ after the sample extraction from the Rn-box.

The measurement that have been carried out are sensitive just to those isotopes that decay through the alpha channel. This implies that there is not any direct information about the presence of ${ }^{214} \mathrm{~Pb}$ and ${ }^{214} \mathrm{Bi}$ contaminations on the samples. These two isotopes are, together with ${ }^{218} \mathrm{Po},{ }^{214} \mathrm{Po}$ progenitors. Studying the variation in time of ${ }^{214} \mathrm{~Pb}$ and ${ }^{214} \mathrm{Bi}$ activities it is possible to infer the initial ${ }^{214} \mathrm{~Pb}$ and ${ }^{214} \mathrm{Bi}$ contaminations on the samples. In order to evaluate the activities of the various elements of the chain, the Bateman's equations have been solved [17].

The values are the following:

$$
\begin{aligned}
& A_{214}^{0} \mathrm{~Pb}=0.12 \pm 0.04 \mathrm{~Bq} \mathrm{~cm}^{-2} \\
& A_{214}^{0} \mathrm{Bi}=0.09 \pm 0.06 \mathrm{~Bq} \mathrm{~cm}^{-2} \\
& A_{214}^{0} \mathrm{Po}=0.12 \pm 0.04 \mathrm{~Bq} \mathrm{~cm}^{-2}
\end{aligned}
$$

the activities comparison for the various elements shows that the Radon chain, when the samples were extracted from the Rn-box, was at the equilibrium between the ${ }^{218} \mathrm{Po}$ and the ${ }^{214} \mathrm{Po}$. The consequence is that the main contaminant that sticks on the samples is ${ }^{218} \mathrm{Po}$, that generates its own chain on the sample producing $\mathrm{Pb}$ and $\mathrm{Bi}$ isotopes. Indeed if ${ }^{214} \mathrm{~Pb}$ or ${ }^{214} \mathrm{Bi}$ had stuck on the samples, then the chain would have not been at the equilibrium, but we would have observed a higher contamination for these elements that have half-lives much longer than ${ }^{218} \mathrm{Po}$. The same argument can be adopted for ${ }^{214} \mathrm{Po}$, but in that case it would be impossible to detect such an excess, because of its short half-life.

Finally, on Fig. 2 we observe a signal at $5.3 \mathrm{MeV}$ which is produced by the alpha decay of ${ }^{210} \mathrm{Po}$. We have demonstrated that ${ }^{214} \mathrm{~Pb}$ does not stick on the sample, and assuming the sticking process to be a chemical one (therefore identical for all the isotopes of the same element), we can conclude that ${ }^{210} \mathrm{~Pb}$ does not stick. Therefore the ${ }^{210} \mathrm{Po}$ initial activity is produced just by its sticking on the samples, hence by the ${ }^{210}$ Po concentration inside the Rn-box.

According to our picture while inside the Rn-box the Polonii isotopes stick on the sample a re-generation of the radioactive chain is produced. However while secular equilibrium is easily restored between the fast decaying isotopes,

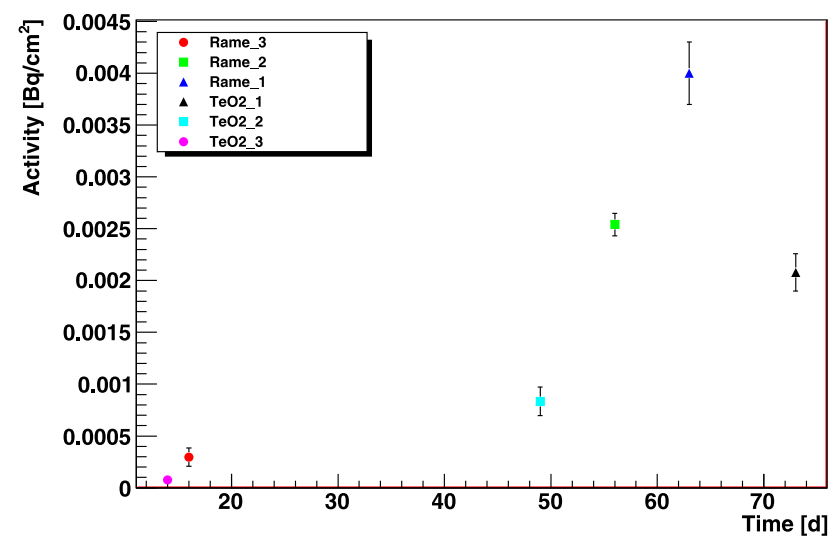

Fig. $5{ }^{210} \mathrm{Po}$ activity of copper and tellurium samples as a function of the ${ }^{222} \mathrm{Rn}$ exposure time

${ }^{210} \mathrm{~Pb}$ and ${ }^{210} \mathrm{Po}$ are not in equilibrium. After the extraction of the sample from the Rn-box the ${ }^{210}$ Po production is driven by two different mechanisms: the first one is the direct production of ${ }^{210} \mathrm{Po}$ from the decay of ${ }^{210} \mathrm{~Pb}$ isotopes stuck on the sample during its exposure to ${ }^{222} \mathrm{Rn}$ the second one is due to the regeneration of ${ }^{210} \mathrm{~Pb}$ from the continuous decay of the parent nuclei (e.g. ${ }^{218}$ Po and its daughters). As a consequence after the extraction from the Rn-box, the ${ }^{210} \mathrm{Po}$ is slowly (almost linearly) increasing its activity, trying to reach the equilibrium with its parent: ${ }^{210} \mathrm{~Pb}$.

All the exposed samples show the same behavior for the activity of the Rn daughters, but different absolute values depending whether they are made of copper or of tellurium dioxide. The reason for the different value of the contaminations are related to the chemical features of materials: copper is more chemically active than tellurium oxide.

${ }^{210} \mathrm{Po}$ activities measured on copper and tellurium dioxide samples, after the exposure, are reported in Fig. 5 as a function of Rn exposure. We can see that the relation between exposure and ${ }^{210} \mathrm{Po}$ activity seems to be slightly different from the linear regime, this effect is caused by the fact that inside the Rn-box the secular equilibrium, among the element of the Rn-chain, is still not reached (e.g. ${ }^{210} \mathrm{Po}$ ).

\section{Diffusion of contaminants}

In the previous section we showed that the sticking elements are only the Polonii isotopes. These, once bounded to the surface, start to decay and produce a regeneration of a part of the Rn chain inside the sample. In this section we discuss another important feature of the contamination: its diffusion inside the material. The density profile of the radioimpurity distribution is particularly important to evaluate the impact that the contaminations may have on the detector counting rate. 
Figure 2 shows that the Polonium contamination is not strictly superficial but it has a deep profile inside the matrix, clearly observable in the asymmetric component of the ${ }^{214} \mathrm{Po}$ and ${ }^{210} \mathrm{Po}$ signals. In the following analysis the correlation between the contamination profile and the time exposure to Radon is investigated.

Focusing on ${ }^{210} \mathrm{Po}$, which is the alpha emitter in the Radon chain with the longest half-life (therefore the most dangerous for experiments). We reconstruct the ${ }^{210} \mathrm{Po}$ energy spectrum using Monte Carlo simulations, based on GEANT4 code [18], in order to understand the contamination profile. Assuming that the alpha peak at $5.3 \mathrm{MeV}$ and its tail are caused by two different processes: a deposition and a diffusion of the contaminants. The effect of the deposition is the creation of a superficial distribution of contaminant that was reproduced in the simulation with a uniformly contaminated layer thick $0.5 \mathrm{~nm}$ (this depth gives a spread which is much smaller than detector resolution), the effect of the diffusion is the creation of a deep contamination that was described in the simulation with a layer having an exponential profile inside the sample [15].

The diffusion profiles are reconstructed with relative accuracy (see Fig. 6). In Table 1 the diffusion depths for the samples are reported. The evaluation of errors on the diffusion depths is given by the detector resolution which limits the accuracy achievable in the profile description to $20 \mathrm{~nm}$.
The former statement is validated by comparing the diffusion depth of ${ }^{210} \mathrm{Po}$ with that obtained for ${ }^{214} \mathrm{Po}$. The diffusion depth of the latter nuclide does not change with $\mathrm{Rn}$ exposure but it has a fixed value $(380 \pm 20 \mathrm{~nm}$ for copper and $520 \pm 20 \mathrm{~nm}$ for tellurium dioxide). The reason for this behavior is the short half-life of this isotope $\left(T_{1 / 2}=0.164 \mathrm{~ms}\right)$ which is several orders of magnitude smaller than the exposure time of the samples, and does not have enough time for generating a deep diffusion.

The tellurium dioxide samples have a deeper contamination compared to the copper ones. The grinding of the surface is the reason for this behavior. The crystal structure is highly regular in the tellurium, allowing a diffusion with less impedance, compared to the copper one.

Table 1 Diffusion length of ${ }^{210} \mathrm{Po}$

\begin{tabular}{lllc}
\hline Sample & Material & Exposure & Diffusion depth \\
\hline Copper_1 & $\mathrm{Cu}$ & $63 \mathrm{~d}$ & $420 \pm 20 \mathrm{~nm}$ \\
Copper_2 & $\mathrm{Cu}$ & $56 \mathrm{~d}$ & $360 \pm 20 \mathrm{~nm}$ \\
Copper_3 & $\mathrm{Cu}$ & $16 \mathrm{~d}$ & $20 \pm 20 \mathrm{~nm}$ \\
$\mathrm{TeO} 2 \_1$ & $\mathrm{TeO}_{2}$ & $73 \mathrm{~d}$ & $940 \pm 20 \mathrm{~nm}$ \\
$\mathrm{TeO} 2 \_2$ & $\mathrm{TeO}_{2}$ & $49 \mathrm{~d}$ & $480 \pm 20 \mathrm{~nm}$ \\
$\mathrm{TeO} 2 \_3$ & $\mathrm{TeO}_{2}$ & $14 \mathrm{~d}$ & $60 \pm 20 \mathrm{~nm}$ \\
\hline
\end{tabular}

Fig. 6 Reconstruction of the two components (peak and tail) ${ }^{210}$ Po signal (top) for the copper sample Copper_1 (diffusion profile $430 \mathrm{~nm}$ ) and (bottom) for the tellurium one $\mathrm{TeO} 2 \_2$ (diffusion profile $940 \mathrm{~nm}$ )
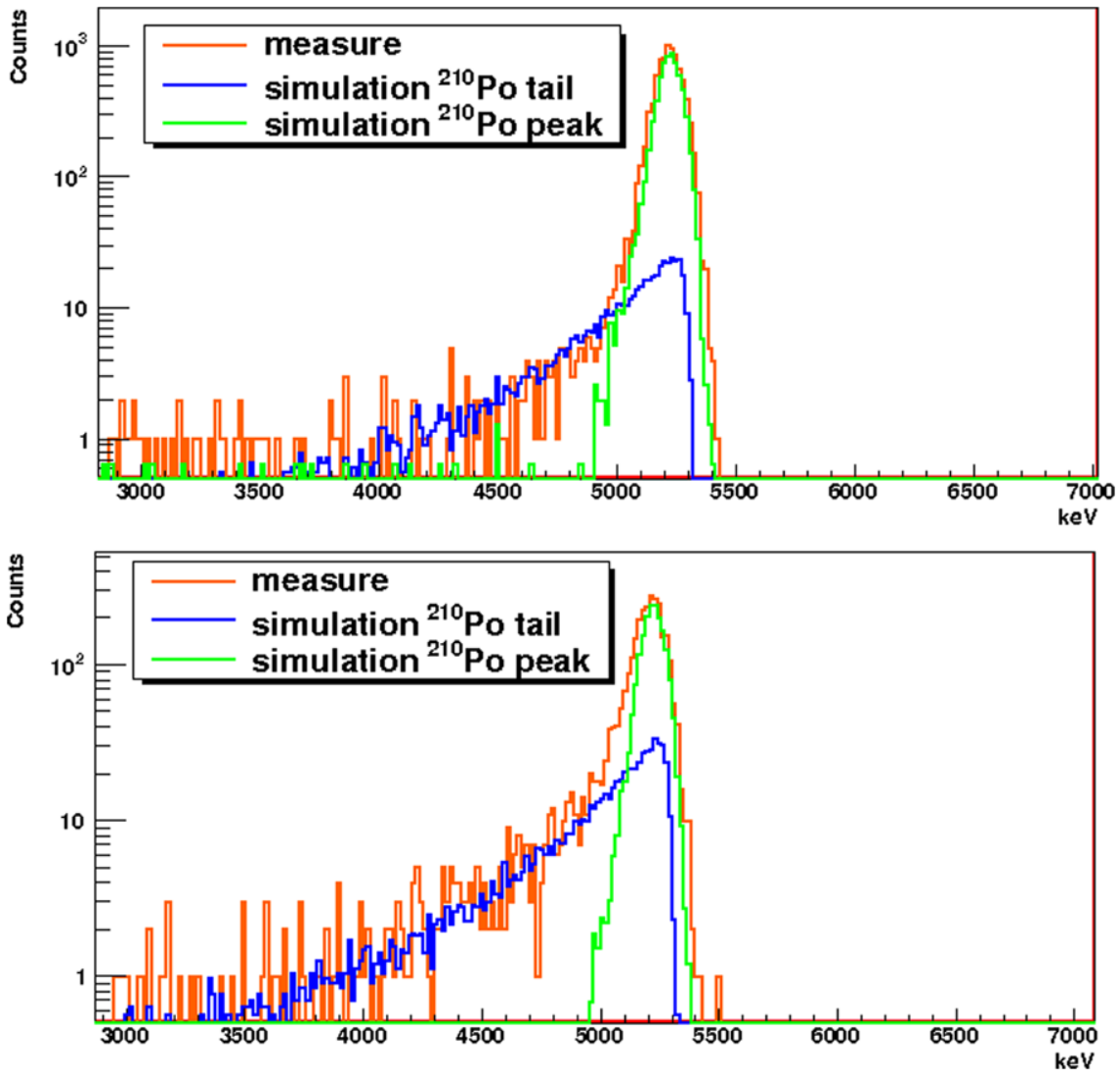
The ${ }^{218} \mathrm{Po}$ signal is the one with the poorest statistics among the other isotopes. This makes the evaluation of the asymmetric component of signal much tricky. In order to evaluate the asymmetry of the peak the skewness parameter is used, which is an estimator of the degree of asymmetry of a distribution [19]. The skewness estimator of a distribution is defined as [20]:

$g_{1}=\frac{k_{3}}{k_{2}^{3 / 2}}$

where $k_{i}$ is the $i$ th central moment. The skewness for the ${ }^{218} \mathrm{Po}$ signal is $0.40 \pm 0.20$, the peak is Gaussian at a confidence level of $90 \%$.

The implantation depth of ${ }^{222} \mathrm{Rn}$ nuclear recoil in copper ${ }^{218} \mathrm{Po}$ is about $15 \mathrm{~nm}$ (evaluated with Monte Carlo simulation based on the [16] code), not computable from our measurements due to the limited resolution of the detector. This explains the symmetry of the ${ }^{218}$ Po signal in our data. The surface implantation of ${ }^{218} \mathrm{Po}$ induces deeper ${ }^{214} \mathrm{Po}$ and ${ }^{210} \mathrm{Po}$ contaminations, that depends on the isotope half-lives and on the exposure of the sample to ${ }^{222} \mathrm{Rn}$. Direct $\mathrm{Pb}$ contaminations give just a small contribution to the overall contamination of the sample. Furthermore, a mild surface cleaning (ultra-pure water and isopropyl alcohol) reduced negligibly the overall contamination of the sample; ${ }^{210} \mathrm{~Pb}$ deposition on the sample is a second order process.

\section{Radon sticking factor: definition}

In this section we evaluate the Rn sticking factor defined in Table 2. Let's assume to have a surface $(S)$ exposed to a high Radon concentration atmosphere $(n=$ Radon nuclei per unit volume). To compute the number of Radon nuclei which hit the surface per time unit and surface unit (e.g. the flux), $\Gamma$, we need to consider the number of nuclei in the volume $v \cdot t$ over the sample's surface, where $v$ is the particle velocity (at a temperature of $300 \mathrm{~K}$ ) and $t$ is the time unit. If we integrate the solid angle in which all the particles in the volume $v \cdot t$ see the surface $S$, we obtain:

$$
\begin{aligned}
& \Gamma\left[\frac{\text { hits }}{\mathrm{cm}^{2} \mathrm{~s}}\right] \\
& \quad=\int_{0}^{2 \pi} d \phi \int_{0}^{\frac{\pi}{2}} d \theta \sin \theta \int_{0}^{v} d r r^{2}\left(\frac{S \cos \theta}{4 \pi r^{2}}\right) \frac{n}{S} \\
& \quad=\frac{n \cdot v}{4} .
\end{aligned}
$$

The thermodynamics allows to define the velocity of a nucleus at a given temperature as:

$v=\sqrt{\frac{k_{b} T}{m}}$
Table 2 Radon sticking factor value for different materials

\begin{tabular}{llll}
\hline Sample & Material & Exposure & $\Sigma_{\mathrm{Rn}}$ \\
\hline Copper_1 & $\mathrm{Cu}$ & $63 \mathrm{~d}$ & $1.86 \cdot 10^{-9} \pm 1.01 \cdot 10^{-10}$ \\
Copper_2 & $\mathrm{Cu}$ & $56 \mathrm{~d}$ & $6.99 \cdot 10^{-10} \pm 1.82 \cdot 10^{-11}$ \\
Copper_3 & $\mathrm{Cu}$ & $16 \mathrm{~d}$ & $5.16 \cdot 10^{-10} \pm 2.78 \cdot 10^{-11}$ \\
$\mathrm{TeO} 2 \_1$ & $\mathrm{TeO}_{2}$ & $73 \mathrm{~d}$ & $3.75 \cdot 10^{-10} \pm 2.10 \cdot 10^{-11}$ \\
$\mathrm{TeO} 2 \_2$ & $\mathrm{TeO}_{2}$ & $49 \mathrm{~d}$ & $3.13 \cdot 10^{-10} \pm 1.19 \cdot 10^{-11}$ \\
$\mathrm{TeO} 2 \_3$ & $\mathrm{TeO}_{2}$ & $14 \mathrm{~d}$ & - \\
\hline
\end{tabular}

where $k_{b}$ is the Boltzmann's constant, $m$ the mass of the nucleus and $T$ the temperature. The Radon concentration of $315 \mathrm{kBq} \mathrm{m}^{-3}$ gives:

$\Gamma=5.85 \cdot 10^{9} \frac{\text { hits }}{\mathrm{cm}^{2} \mathrm{~s}}$

Once we have the total number of nuclei which have a not vanishing probability to hit on (hence to stick) the slab, the computation is almost done. The number of nuclei which has stuck on the slab is:

$N=A_{\mathrm{Pb}}^{0} \cdot \tau_{\mathrm{Pb}}$

where $A_{\mathrm{Pb}}^{0}$ is the ${ }^{210} \mathrm{~Pb}$ activity measured at the saturation $\left({ }^{210} \mathrm{~Pb}\right.$ and ${ }^{210} \mathrm{Po}$ are at the equilibrium). We refer to the ${ }^{210} \mathrm{~Pb}$ activity because we assume that after a long period of time $\left(t \gg \tau_{\mathrm{Rn}}\right.$ ), all the ${ }^{222} \mathrm{Rn}$ daughters have decayed and have populated the ${ }^{210} \mathrm{~Pb}$ level of the Rn decay-chain. We consider the ${ }^{210} \mathrm{~Pb}$ as an "integrator" of all the nuclei that have stuck on the surface $\left({ }^{218} \mathrm{~Pb}\right.$ and $\left.{ }^{214} \mathrm{Po}\right)$.

$A_{\mathrm{Pb}}^{0}$ is derived from the equation:

$A_{\mathrm{Po}}=A_{\mathrm{Pb}}^{0} \frac{\lambda_{\mathrm{Pb}}}{\lambda_{\mathrm{Po}}-\lambda_{\mathrm{Pb}}}\left(e^{-\lambda_{\mathrm{Pb}} t}-e^{-\lambda_{\mathrm{Po}} t}\right)$

$\mathrm{A}_{\mathrm{Po}_{\mathrm{o}}}$ is the activity of the sample measured after its exposure to $\mathrm{Rn}\left(t \gg \tau_{\mathrm{Rn}}\right.$, in our case $\left.t \sim 1 \mathrm{y}\right)$, and $\lambda_{X}$ is the mean half-life of the $\mathrm{X}$ element.

Finally the sticking factor is computed using the following formula:

$\Sigma_{\mathrm{Rn}}=\frac{A_{210 \mathrm{~Pb}}^{0} \cdot \tau_{210} \mathrm{~Pb}}{\Gamma \cdot S \cdot t_{\mathrm{exp}}}$

where $S$ is the slab surface and $t_{\exp }$ the time exposure.

After a thorough analysis we can state that the Radon sticking factor of the studied materials has small values for a high ${ }^{222} \mathrm{Rn}$ concentration. The sticking factor of the samples which had a smaller time exposure will tend to the value of the ones which were exposed for longer time.

The copper samples being more chemically reactive (high electronegativity) show a higher Radon sticking factor compared to the tellurium dioxide ones. 


\section{Conclusions}

According to the results of our study the exposure of detector components to a ${ }^{222} \mathrm{Rn}$ contaminated atmosphere has as a final effect the creation of a long-living contamination of ${ }^{210} \mathrm{~Pb}$ and its progenies. The contamination is due to Polonii isotopes that stick on the sample, the longer is the Rn exposure the higher is the contamination and the deeper is the distribution of long-living isotopes. The latter feature is quite relevant because the density profile of the contamination can greatly influence the real impact on the background counting rate of the experiment.

Open Access This article is distributed under the terms of the Creative Commons Attribution Noncommercial License which permits any noncommercial use, distribution, and reproduction in any medium, provided the original author(s) and source are credited.

\section{References}

1. V.I. Tretyak, Yu.G. Zdesenko, At. Data Nucl. Data Tables 80, 83 (2002)

2. S. Elliott, P. Vogel, Annu. Rev. Nucl. Part. Sci. 52, 115 (2002)
3. A. Morales, J. Morales, Nucl. Phys. B, Proc. Suppl. 114, 141 (2003)

4. F.T. Avignone III, S.R. Elliott, J. Engel, Rev. Mod. Phys. 80, 482 (2006)

5. G. Jungman, M. Kamionkowski, K. Griest, Phys. Rep. 267, 195 (1996)

6. J.R. Primack, B. Sadoulet, D. Seckel, Annu. Rev. Nucl. Part. Sci. 38, 751 (1988)

7. P.F. Smith, J.D. Lewin, Phys. Rep. 187, 203 (1990)

8. G. Bertone, D. Hooper, J. Silk, Phys. Rep. 405, 279-390 (2005)

9. G. Heusser, Annu. Rev. Nucl. Part. Sci. 45, 543-590 (1995)

10. D.S. Leonard, P. Grinberg et al., Nucl. Instrum. Methods Phys. Res., Sect. A, Accel. Spectrom. Detect. Assoc. Equip. 591, 490509 (2008)

11. C. Bucci et al., Eur. Phys. J. A 41, 155 (2009)

12. E. Armengaud et al., Phys. Lett. B 687, 294-298 (2010)

13. C. Arnaboldi et al., Nucl. Instrum. Methods Phys. Res., Sect. A, Accel. Spectrom. Detect. Assoc. Equip. 518, 775 (2004)

14. R. Ardito et al., CUORE: a cryogenic underground observatory for rare events (2005). arXiv:hep-ex/0501010

15. F. Ribeiro et al., Radiat. Meas. 26, 747-750 (1996)

16. J. Ziegler et al., SRIM, The stopping and range of ions in matter (2008)

17. J. Cetnar, Ann. Nucl. Energy 33, 640-645 (2006)

18. S. Agostinelli et al., Nucl. Instrum. Methods Phys. Res., Sect. A, Accel. Spectrom. Detect. Assoc. Equip. 506, 250-303 (2003)

19. J.F. Kenney, E.S. Keeping, Math. Stat. (1951)

20. J.F. Kenney, E.S. Keeping, J. Math. Stat. 1, 252-285 (1962) 